\title{
Response to safety and long-term efficacy of repeated dexamethasone intravitreal implant for the treatment of cystoid macular edema secondary to retinal vein occlusion with and without a switch to anti-VEGF agents: a 3-year experience
}

\author{
Julie Blanc $^{1}$ - Laurent Kodjikian ${ }^{2} \cdot$ Alain Bron ${ }^{1}$ (D) $\cdot$ Catherine Creuzot-Garcher ${ }^{1}$
}

Published online: 6 August 2018

(C) Springer-Verlag GmbH Germany, part of Springer Nature 2018

Dear Editor,

We thank our colleagues Dan Călugăru and Mihai Călugăru for their interest in our paper recently published in this Journal [1].

We thank them for highlighting their contribution to retinal vein occlusion (RVO) through their own published papers. They highlighted some weaknesses in our report that were acknowledged in the section limitations of the study in the Discussion.

They mention that a washout period should have been respected when switching to anti-VEGF agents. This multicenter study had no other ambition to show what is really done in clinical practice, the so-called real world, so often mentioned in the literature. In fact, the value of prospective studies with well-defined definitions and protocol details is not debatable. This is the basis for the evaluation of different treatment modalities. However as has already been pointed out "The paradox of the clinical trial is that it is the best way to assess whether an intervention works, but it is arguably the worst way to assess who will benefit from it." [2]

Therefore, we need both kind of studies, and these real-life studies are constantly leading to poorer results than the good RCTs dealing with "perfect patients" attending the visits, taking the medication and fulfilling the inclusion and exclusion criteria. For clinicians, the additional challenge is to take care of less perfect patients, and this is really valuable, bringing other information.

It is however reassuring to note that our colleagues in spite of advocating the need to adhering to strict definitions, protocols etc. do have some beliefs: "We believe that the front-line therapy for the treatment of ME associated with RVOs is represented by the specific anti-VEGF drugs (bevacizumab/ranibizumab/aflibercept)."

We do respect the beliefs of our colleagues. The debate between the pro and cons of anti-VEGF agents or dexamethasone in the management of RVO remains open and we can easily find in the literature strong arguments to promote one treatment or the other. Our aim was not to support any treatment, but to report on the treatment carried out in clinical practice. We do think that instead of opposing the two treatment modalities, we should consider how fortunate we are nowadays to have several treatments to fight against RVO, a sight-threatening condition.

The aims of our treatments are for the well-being and the quality of life of our patients for these blinding disorders [3].

\section{References}

1. Blanc J, Deschasse C, Kodjikian L et al (2018) Safety and long-term efficacy of repeated dexamethasone intravitreal implants for the treatment of cystoid macular edema secondary to retinal vein occlusion with or without a switch to anti-VEGF agents: a 3-year experience. Graefes Arch Clin Exp Ophthalmol 256:1441-1448

2. Mant D (1999) Can randomised trials inform clinical decisions about individual patients? Lancet 353:743-746

3. Hayreh SS (2005) Prevalent misconceptions about acute retinal vascular occlusive disorders. Prog Retin Eye Res 24:493-519
Alain Bron

alain.bron@chu-dijon.fr

1 University Hospital, Dijon, France

2 University Hospital, Lyon, France 